\title{
MOVILIZACIÓN DE CARBONO ORGÁNICO POR DISTINTOS PROCESOS EROSIVOS EN LA CONEXIÓN LADERA-CAUCE
}

\author{
E. NadeU ${ }^{1}$, C. Boix-Fayos ${ }^{1}$, J. DE Vente ${ }^{2}$, J. LÓPeZ ${ }^{1} \&$ M. MartíneZ-Mena ${ }^{1}$ \\ 1 Departamento de Conservación de Suelos y Aguas y Manejo de Residuos Orgánicos, CEBAS-CSIC, \\ Campus Universitario de Espinardo, Apartado de Correos 164, 30100 Murcia, España \\ 2 Departamento de Desertificación y Geoecología, EEZA-CSIC, General Segura 1, \\ 04001 Almería, España \\ E-mail: enadeu@cebas.csic.es
}

\begin{abstract}
With the purpose of analysing the type (labile or stable) and quantity of organic carbon (OC) mobilized by different erosive processes identified at the slope-bed connection, the erosion deposits of gullies, sheet erosion, bank erosion and tillage erosion were studied in a small catchment (10 ha) and compared to the characteristics of the catchment soils. Selectivity upon soil detachment and transport was associated to different $O C$ content and types in the erosion deposits. Enrichment ratios of organic carbon sediment/soil were low $(\sim 0,40 \pm 0,26)$, even though a slight enrichment was described for fine particles (positively correlated to CO). These results were attributed to mineralization processes prevailing over OC burial in a very active channel where depositional sites are scarce.
\end{abstract}

Keywords: Erosion processes; soil organic carbon; enrichment ratio; catchment; slope-bed connection.

RESUMEN.- Con el fin de caracterizar la cantidad y tipo (lábil o recalcitrante) de carbono orgánico (CO) movilizado por distintos procesos erosivos identificados en las conexiones ladera-cauce, se estudiaron las características de los depósitos de erosión concentrada en cárcavas, erosión hídrica laminar, erosión lateral-gravitacional y erosión por laboreo en el contacto ladera-cauce de una cuenca de pequeño tamaño (10 ha) y se relacionaron con las características de los suelos-fuentes originales de donde procedian. La selectividad en el arranque y transporte de suelo de los distintos procesos se pudo asociar a diferentes contenidos y tipos de $\mathrm{CO}$ en los depósitos. Las razones de enriquecimiento de carbono orgánico sedimento/suelo fueron bajas $(\sim 0,40 \pm 0,26)$, a pesar de haber un ligero enriquecimiento en partículas finas (correlacionadas positivamente con el CO) en los depósitos. Todo ello se atribuyó a los efec- 
E. NADEU, C. BOIX-FAYOS, J. DE VENTE, J. LÓPEZ \& M. MARTÍNEZ-MENA

tos de la mineralización en un cauce muy activo con pocas zonas de deposición y abundantes procesos de erosión no selectiva.

Palabras clave: Procesos erosivos; carbono orgánico del suelo; razón de enriquecimiento; cuenca, conexión ladera-cauce.

\section{Introducción}

La importancia de la erosión del carbono orgánico (CO) a escala de cuenca, en el contexto actual de cambio climático, radica en la cantidad de CO que puede quedar secuestrada o por el contrario ser liberada a la atmósfera durante y debido a los procesos erosivos y qué parte representa éste $\mathrm{CO}$ movilizado en el conjunto del ciclo del carbono terrestre. Acerca de la primera cuestión existen dos posicionamientos en la comunidad científica: (i) la erosión favorece la mineralización de carbono y (ii) los procesos erosivos contribuyen a su secuestro. Los científicos que respaldan la primera postura (LAL, 2001; LAL, 2003; ZHANG et al., 2006) han estimado tasas de emisión de carbono a escala global de 0,4-1,4 Pg/año de los suelos a la atmósfera debido a la erosión, mientras que el otro grupo (LIU et al., 2003; SMITH et al., 2001; STALLARD, 1998; VAN OOST et al., 2005; YOO, 2005) ha calculado tasas de secuestro asociadas a procesos erosivos entre 0,55 y $1 \mathrm{Pg} /$ año, y hasta con capacidad para compensar un $10 \%$ de las emisiones de combustibles del año 2005 (BERHE et al., 2007).

Como bien observaron BERHE et al. (2007), el desacuerdo entre científicos se produce al centrarse cada grupo en partes distintas del proceso de erosión. De este modo, mientras unos tienen como objeto de estudio la tasa de mineralización del CO al ser arrancado, transportado y depositado, para los otros la deposición del CO y su reemplazo en las laderas erosionadas es el centro de atención.

Finalmente, hay que tener en cuenta que aunque a nivel de ecosistema la pérdida de carbono por erosión se estime en solo el $1 \%$ de la productividad primaria neta (PPN) (SCHLESINGER \& MELACK, 1981) y que haya otros factores causantes de la pérdida de $\mathrm{CO}$ con más relevancia -entre ellas las prácticas agrícolas (OWENS et al., 2002)-, es importante resaltar que su contribución relativa en ecosistemas con suelos degradados y muy bajos niveles de materia orgánica, como es común en áreas semiáridas, es notable (MARTÍNEZ-MENA et al., 2008), así como en suelos con bajos niveles de carbono pero moderados niveles de erosión, pudiendo tener un impacto importante en el balance de carbono (QUINTON et al., 2006). 


\section{Movilización del carbono por erosión hídrica: cuestiones de escala y mecanismos operantes}

La erosión de los suelos es un fenómeno complejo con una gran dependencia de la escala espacial y temporal. Espacialmente se ha demostrado en los últimos años que los procesos a escalas más finas de observación (escalas mayores) implican el arranque de partículas del suelo, la erosión laminar y la erosión en regueros, mientras que a escalas más gruesas (o escalas más pequeñas) aparecen fenómenos como la erosión en cárcavas, erosión del lecho del río, erosión de los márgenes laterales y movimientos en masa. Por ese motivo, los resultados de estudios de erosión en parcelas no son extrapolables a áreas de mayor superficie.

En las investigaciones realizadas sobre erosión hídrica y carbono, los esfuerzos más grandes se han centrado en la descripción y análisis de los procesos a escala de detalle. Aunque existen numerosos estudios con estimaciones de tasas generales de erosión de suelos y deposición de sedimentos a nivel de cuenca en base a modelos y medidas en campo (QUINTON et al., 2006; SCHIETTECATTE et al., 2008b; WALLING, 1983); sigue habiendo pocas experiencias en el caso del carbono orgánico a escala de cuenca. Sin embargo, ¿es correcto suponer que las distintas fracciones de carbono del suelo responden frente a la erosión hídrica del mismo modo que las partículas minerales? Mientras hay estudios que muestran que en determinadas zonas puede efectivamente existir un patrón común de movimiento del suelo y movilización de CO (RITCHIE et al., 2007), algunas publicaciones apuntan en otra dirección (STRICKLAND et al., 2005).

Hasta el momento, los trabajos realizados coinciden en el hecho de que la erosión es un fenómeno muy selectivo (STOLTENBERG \& WHITE, 1953; GREGORICH et al., 1998) que moviliza el carbono orgánico (CO) e inorgánico de modo distinto afectando los componentes superficiales (ROOSE, 2006) más pequeños y de menor densidad. De este modo, y debido a su baja estabilidad y a su alta concentración en los horizontes superficiales, el CO del suelo es fácilmente movilizado y se puede acumular en los sedimentos a mayores concentraciones que en los suelos de donde procede. Para valorar estas diferencias, se define una razón de enriquecimiento (RE) que toma valores por encima de uno cuando la concentración de $\mathrm{CO}$ en los sedimentos es mayor que en los suelos originales. Diversos autores (RODRIGUEZ et al., 2004; HAREGEWEYN et al., 2008; RHOTON et al., 2006) han encontrado una buena correlación entre el tamaño de partículas y la razón de enriquecimiento, siendo las de menor tamaño (arcilla y limo) las que presentan los valores más elevados. 
Otras investigaciones apuntan hacia una diferenciación en la movilización de $\mathrm{CO}$ según el proceso erosivo ocurrente. Así, la erosión hídrica laminar moviliza sobre todo partículas de pequeño tamaño y superficiales (ROOSE $e t$ al., 2006), lo que originará RE por encima de uno, pudiendo aumentar este coeficiente en eventos con escorrentías débiles donde se erosionan principalmente los elementos más finos del suelo. En la misma línea, en una pequeña cuenca agrícola, OWENS et al. (2002) cuantificaron RE mayores cuando los sedimentos procedían de los primeros $5 \mathrm{~cm}$ de suelo. Las cárcavas, por otro lado, movilizan cantidades muy grandes de material pero una vez agotado el más superficial las partículas potencialmente erosionables son las subsuperficiales, más pobres en $\mathrm{CO}$. Al considerarse la erosión en regueros como no selectiva (SCHIETTECATTE et al., 2008a) parece lógico esperar que la RE en éstos o en cárcavas - al ser éstas también producto de erosión concentrada a una escala superior- sea menor que $1 \mathrm{o}$, al menos, menor que el valor de la razón de la erosión laminar, pues afecta a más profundidad del perfil y aparece una menor selección de las partículas finas del suelo.

Según el tiempo de permanencia en el suelo, se puede distinguir entre $\mathrm{CO}$ particulado (COP) constituido por parte del CO activo (tiempo de permanencia de horas a meses) y del CO lento (años a décadas) y CO mineral asociado (COM) constituido por el $\mathrm{CO}$ lento y el pasivo (con un tiempo de permanencia de siglos a milenios). Estas fracciones se movilizan de manera diferente; al ser más lábil, el COP es transportado con mayor facilidad por la escorrentía superficial o el viento (LAL, 2002). Esta preferencia por la fracción lábil podría significar que los depósitos de sedimentos movilizados por el agua tuvieran razones de enriquecimiento mayores que aquellos movilizados por la erosión por laboreo (ZHANG et al., 2006). Sin embargo, es interesante destacar que MARTÍNEZ-MENA et al. (2008) encontraron que las formas estables de CO (COM) tenían una mayor contribución al carbono total en sedimentos de erosión hídrica que las formas más lábiles (COP) en parcelas experimentales. Concluyeron que la reducción de la fracción de COP en un suelo agrícola comparado con un suelo forestal estaba más ligada al cambio de uso que al propio proceso de erosión hídrica

Por último, aunque a nivel de cuenca existan pocos estudios experimentales, y la mayoría en cuencas agrícolas, los resultados encontrados en otros trabajos muestran que los mecanismos operantes a nivel de parcela no sólo no son válidos para las cuencas sino que pueden llevar a conclusiones erróneas. Por ejemplo, en una cuenca agrícola, las prácticas de manejo del suelo, muy importantes a nivel de parcela, pasan a un segundo término por detrás de los procesos hidrodinámicos, las características de las lluvias o la topografía (JACINTHE et al., 2004; WEI et al., 2008). SMITH et al. (2007) mostraron en una cuenca experimental de $4.900 \mathrm{~km}^{2}$, que mientras en las 
laderas la erosión se mantenía y representaba un $2 \%$ de la PPN, había también grandes áreas de deposición (20\% de la superficie de la cuenca) que equivalían a su vez al 10\% de la PPN. Observaron también que las tasas erosivas eran mayores en campos agrícolas. Por otro lado, aunque se ha comprobado en varios estudios que el balance de sedimentos varía con el tamaño de la cuenca (DE VENTE et al., 2007), y que en algunos casos se ha encontrado una correlación negativa entre el porcentaje de CO erosionado y el área de la cuenca de drenaje (BOIX-FAYOS et al., 2009), otros trabajos no encontraron relación entre estos factores (AVNIMELECH \& MCHENRY, 1984). Además, hay que tener en cuenta que el enriquecimiento de carbono en el sedimento no sólo es causado por la selectividad de la erosión, sino que puede originarse durante la sedimentación al depositarse las partículas gruesas con mayor rapidez que las pequeñas, aunque el primer proceso parece tener una mayor importancia que el segundo (SCHIETTECATTE et al., 2008b). A nivel de cuenca se puede decir que la erosión afecta al balance de $\mathrm{CO}$ al cambiar la magnitud de las entradas de $\mathrm{CO}$ y su descomposición y estabilización (BERHE et al., 2007).

\section{Objetivos}

Partiendo de la hipótesis de que en las conexiones ladera-cauce, la cantidad y tipo de $\mathrm{CO}$ en los depósitos sedimentarios de diferentes procesos erosivos refleja la propia dinámica del proceso, según afecte a la parte superficial o a la profundidad del perfil, se pretende: (i) Conocer la concentración y los tipos de carbono movilizados por distintos procesos erosivos ; (ii) calcular el enriquecimiento para cada fracción de CO (COP y $\mathrm{COM}$ ) en los sedimentos y (iii) establecer una relación entre los reservorios de $\mathrm{CO}$ y la textura en suelos y sedimentos. Con todo ello se pretende aportar nueva información sobre los procesos de erosión de $\mathrm{CO}$ a escala de cuenca.

\section{Materiales y métodos}

\subsection{Zona de estudio}

El barranco muestreado (UTM: 567726.75 X y 4221955.14 Y) se encuentra en la parte baja de la cuenca de la Rogativa $\left(47.2 \mathrm{~km}^{2}\right)$ situada en el NO de la Región de Murcia (SE español, 38 $08^{\circ} \mathrm{N}, 2^{\circ} 13^{\prime} \mathrm{W}$ ) (Fig. 1). La precipitación y 
temperatura media anual es de $583 \mathrm{~mm}$ y $13.3^{\circ} \mathrm{C}$. La litología dominante en la cuenca son las margas, rocas calcáreas, margas carbonatadas y areniscas del Cretácico, Oligoceno y Mioceno (IGME, 1978). Las rocas calcáreas se encuentran en las partes superiores mientras que en los fondos de valle dominan las margas. Los suelos se clasifican como Calcaric Cambisols y Calcaric Regosols. En el área de estudio, de 10 ha de extensión, predominan las areniscas. El lecho del cauce tiene $700 \mathrm{~m}$ de longitud y entre $50 \mathrm{~cm}$ y $3 \mathrm{~m}$ de anchura. Se describen tres usos predominantes del suelo: matorral $(64,5 \%)$, en el cual abundan especies como Cystisus reverchonii, Rosmarinus officinalis, Thymus vulgaris y Genista scorpius; bosque $(33,0 \%)$ con Pinus pinaster y Pinus halepensis y cultivos de secano $(2,5 \%)$.

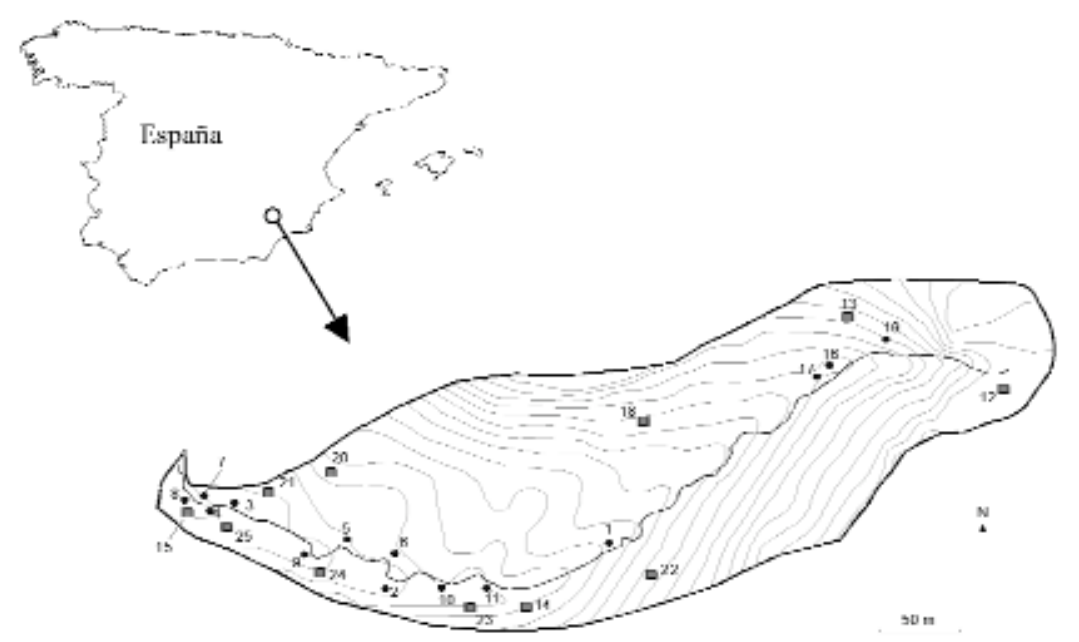

Figura 1. Mapa de situación de la cuenca de estudio. Se indican los lugares de muestreo de suelos (gris) y sedimentos (negro).

Figure 1. Location map for the studied catchment. Sampling sites are indicated as grey squares (soils) and black dots (sediments).

\subsection{Muestreo en campo}

Se realizó un reconocimiento geomorfológico del barranco, basado en la cartografía de fuentes de sedimento desarrollada por Hooke (2003). Con ello se identificaron los principales procesos erosivos en el contacto ladera-cauce. Estos fueron: erosión concentrada en cárcavas, erosión hídrica laminar, erosión lateral y gravitacional, además de erosión por laboreo (Figura 2). En 
la caracterización se realizó una cartografía geomorfológica de algunos tramos que sirvió para profundizar en la dinámica erosiva de las conexioneis ladera-cauce (Figura 3).

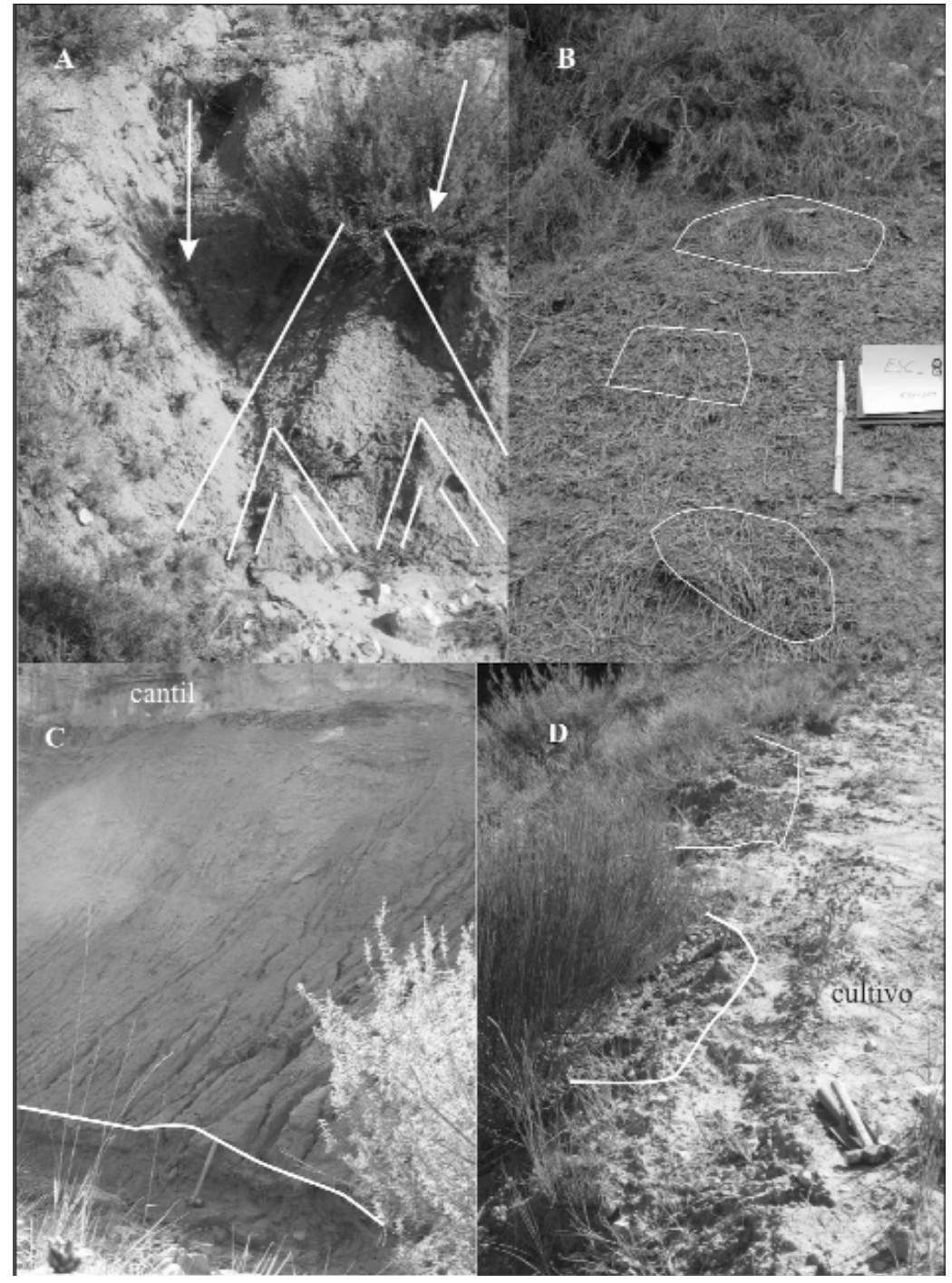

Figure 2. Morfologías erosivas estudiadas. A) Erosión hídrica concentrada en cárcava; B) Erosión hídrica laminar; C) Erosión lateral y gravitacional; D) Erosión por laboreo.

Figure 2. Studied erosion morphologies. A) Gully erosion; B) Interill erosion; C) Bank and gravitational erosion and D) Tillage erosion. 

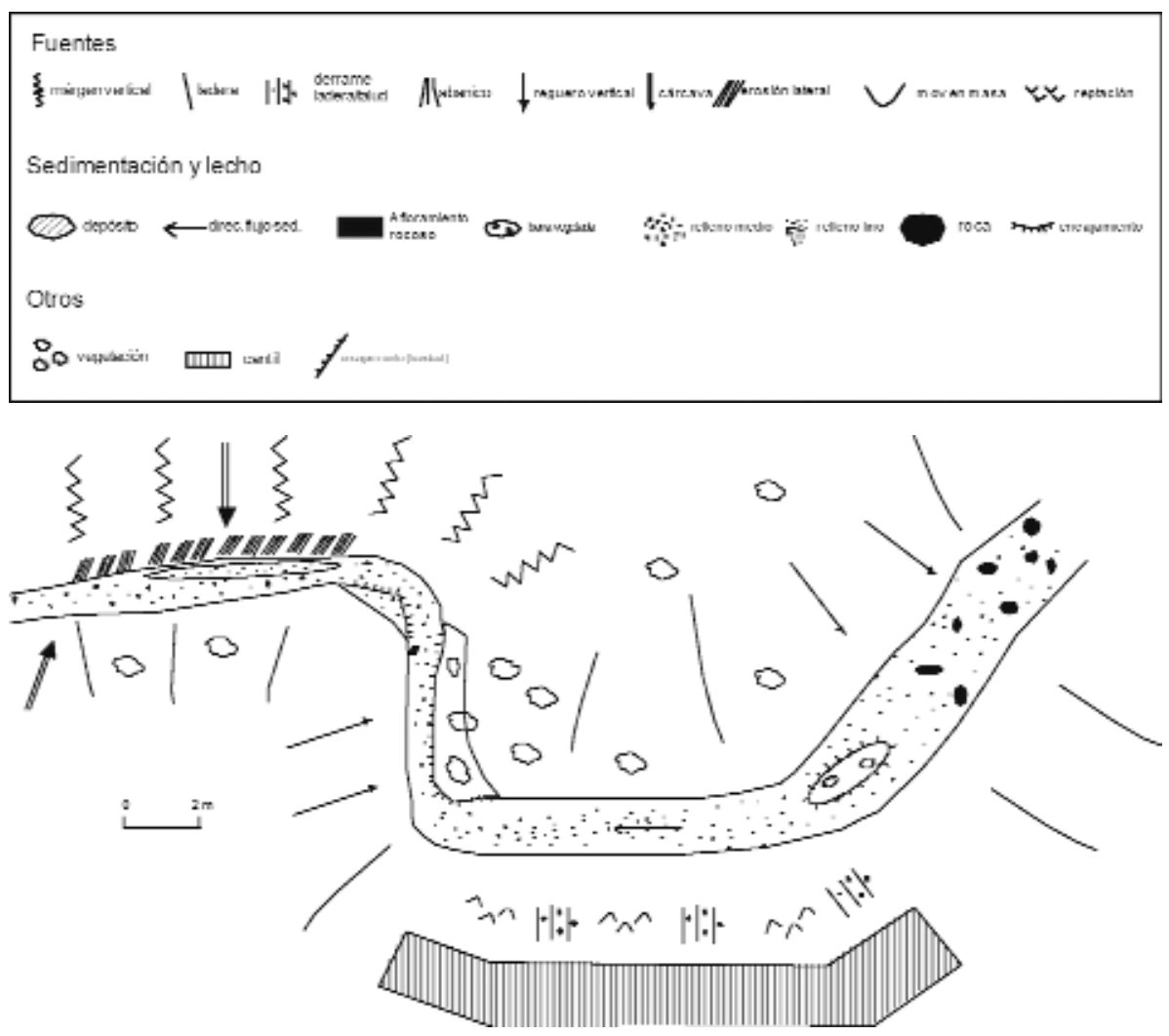

Figura 3. Representación geomorfológica de las conexiones ladera-cauce para un tramo del cauce. Leyenda en la parte superior.

Figure 3. Geomorphological representation of the slope-bed connections for a channel stretch. Legend on top for interpretation.

Se eligieron un mínimo de 2 puntos de muestreo por forma erosiva, aumentando el número de muestras en los casos más recurrentes para mayor representatividad. Se tomaron un total de 32 muestras de sedimentos correspondientes a los 10 primeros centímetros de los depósitos para poder caracterizar su variabilidad. Estas muestras fueron repartidas de la siguiente forma: 14 muestras para depósitos de cárcava, 8 muestras para depósitos de erosión laminar, 6 muestras para depósitos de erosión lateral-gravitacional y 4 muestras para depósitos de erosión por laboreo. En cada punto se tomó una muestra inalterada con un anillo de $100 \mathrm{~cm}^{3}$ para la determinación de la densidad aparente. 
Los suelos se muestrearon acorde a los tres usos principales definidos: bosque, matorral y cultivo. Se tomaron un total de 21 muestras de los primeros $10 \mathrm{~cm}$ entre los 3 usos, descartando la hojarasca y el horizonte orgánico, junto con una muestra inalterada de volumen $100 \mathrm{~cm}^{3}$ para la densidad aparente.

\section{3. Métodos de laboratorio}

Para el análisis de textura las muestras fueron secadas al aire, tamizadas a $2 \mathrm{~mm}$ y posteriormente a $63 \mathrm{~m}$. Las fracciones finas $(<63 \mathrm{~m})$ se analizaron por difracción de láser utilizando un Coulter LS200 tras eliminar la materia orgánica por oxidación y dispersar con calgón. También se dispersaron las arenas $(>63 \mathrm{~m})$ y se separaron $(250 \mathrm{~m})$ por tamizado en húmedo.

La muestra contenida en cada anillo recogido en campo $\left(100 \mathrm{~cm}^{3}\right)$ se dejó secar durante $24 \mathrm{~h}$ a $105^{\circ} \mathrm{C}$ para la determinación de la densidad aparente.

La determinación del carbono orgánico particulado (COP) y el carbono orgánico mineral asociado (COM) se realizó en base al método propuesto por CAMBARDELLA \& ELLIOT (1992). Para ello se tomaron $10 \mathrm{~g}$ de tierra fina $(<2 \mathrm{~mm})$ y se le añadieron $50 \mathrm{ml}$ de hexametafosfato sódico $\left(5 \mathrm{~g}^{-1}\right)$ dejándolos en agitación durante 18 horas para separar las partículas de limo y arcilla y liberar el carbono. A continuación la muestra se tamizó en húmedo con abundante agua destilada recogiendo la fracción inferior en un bote, mientras que la parte superior se filtró con un filtro Whatman 541. Ambas se introdujeron en la estufa a $60-65^{\circ} \mathrm{C}$ hasta estar completamente secas y se pesaron.

Para homogeneizar las fracciones obtenidas, se molieron con un mortero de ágata y se procedió a determinar el contenido en carbono orgánico por oxidación húmeda en medio ácido, con una mezcla caliente de dicromato potásico $1 \mathrm{~N}$ y ácido sulfúrico concentrado según el método de YEOMANS \& BREMNER (1988).

La concentración de carbono orgánico se determinó valorando el exceso de dicromato potásico que no reaccionó mediante una solución ácida de sulfato ferroso amónico (sal de Mohr $0.5 \mathrm{~N}$ ) empleándose para ello un valorador automático Metrohm 785 DMP Titrino.

Para obtener el carbono del suelo en cada una de las dos fracciones ( $\mathrm{g} \mathrm{C}$ $\mathrm{kg}^{-1}$ suelo) se multiplicó el peso seco de cada fracción ( $\mathrm{g} \mathrm{g}^{-1}$ suelo) por la concentración de carbono respectiva $\left(\mathrm{g} \mathrm{C} \mathrm{g}^{-1}\right)$. 


\subsection{Análisis de los datos}

Los análisis estadísticos se realizaron con el paquete SPSS Statistics 17.0. En este trabajo se expresa el valor medio de cada una de las variables analizadas junto con su desviación estándar. Al no presentar los datos una distribución normal ni tener las varianzas homogéneas, se recurrió a la estadística no paramétrica. Los test de Kruskall-Wallis y MannWhitney/Wilcoxon fueron aplicados para encontrar diferencias significativas entre las variables texturales de suelos y sedimentos por separado, así como de $\mathrm{CO}$ en su totalidad y entre los distintos tipos (COP y COM). Para todos los parámetros, se tomó un nivel de significación inferior a 0.05 .

Asimismo, se establecieron correlaciones entre la textura de las muestras y el CO total y sus fracciones. Para ello se utilizó el coeficiente de correlación de Spearman.

\subsection{Cálculo de las razones de enriquecimiento}

Tras la identificación de los procesos erosivos y la realización de la cartografía geomorfológica se constató que había una distribución desigual de los procesos entre los distintos usos del suelo, con lo cual se descartó calcular las tasas de enriquecimiento tomando un único valor medio para muestras de suelo.

El procedimiento realizado parte de las siguientes consideraciones: 1) dado que las muestras de sedimentos de erosión por laboreo se tomaron dentro del barranco pero en los bordes de los campos de cultivo, resulta evidente su procedencia y la razón se calculó en base a los valores medios de los suelos de los campos de cultivo; 2) al presentarse las cárcavas mayoritariamente en el margen derecho y estar este ocupado por matorral, se relacionaron los valores de muestras sedimentarias de cárcava con las medias de los suelos de matorral para la obtención de dichas tasas de enriquecimiento; 3) las tasas correspondientes a las muestras de erosión lateral/gravitacional y laminar se calcularon haciendo un promedio ponderado de los valores de suelos de bosque y matorral en función del porcentaje de distribución de cada uso. 


\section{Resultados y discusión}

\subsection{Reconocimiento geomorfológico}

El reconocimiento geomorfológico en las zonas de contacto ladera-cauce permitió constatar el predominio de formas de erosión no selectivas, que afectan a la totalidad del perfil y hasta el regolito, como son la erosión hídrica concentrada y la erosión lateral. La elevada actividad erosiva del barranco se puso de manifiesto al observarse escasas zonas de deposición de sedimentos. Esto marca la diferencia con procesos erosivos en otros cauces donde sumideros como diques y llanuras y barras aluviales estables facilitan el secuestro de CO (BERHE et al., 2008; BOIX-FAYOS et al., 2009; SMITH et al., 2007). En la Figura 3 se muestra un ejemplo de los esquemas geomorfológicos realizados para el reconocimiento de los procesos dominantes y la caracterización de la dinámica del cauce tanto en las zonas de contacto con la ladera como en el propio lecho.

Además, hubo un contraste marcado entre las dos laderas de la cuenca. En la ladera con orientación solana, recubierta por matorral y que presentaba algunas zonas semi-desnudas, predominaban los procesos de erosión concentrada y erosión lateral. Mientras que en la ladera umbría, recubierta mayoritariamente por bosque de pinos, la erosión por reptación y la erosión gravitacional en los márgenes del cauce eran los procesos más frecuentes.

\subsection{Textura y carbono orgánico en suelos}

Los tres grupos de usos de suelo presentaron una textura media moderadamente gruesa, con la fracción arenas entorno al 50\%, 40\% de limos y $<10 \%$ de contenido en arcillas. Los suelos de bosque fueron los más arenosos $(57 \%)$ y tuvieron valores de arcilla y limo significativamente inferiores a los de las muestras de cultivo y matorral. Por otro lado, no existieron diferencias entre los contenidos en limo grueso o arena fina para las muestras de los distintos suelos.

La concentración de carbono orgánico en el conjunto de muestras de suelo de bosque fue significativamente (p: 0.001) más alta $\left(44,2 \mathrm{~g} \mathrm{~kg}^{-1} \pm 15,1\right)$ que la de los otros dos grupos de suelos $\left(8,75 \mathrm{~g} \mathrm{~kg}^{-1} \pm 3,86\right.$ para matorral y $7,67 \mathrm{~g} \mathrm{~kg}$ ${ }^{1} \pm 1,37$ en suelo de cultivo). Esto ocurrió también en el caso del CO de tipo COP y COM. Mientras la distribución media de carbono por tipos de suelo (29\% de COP y $71 \%$ de COM) fue bastante homogénea entre muestras y semejante a la encontrada en otros estudios realizados en condiciones 
ambientales iguales o similares (MARTINEZ-MENA et al., 2008; BOIX-FAYOS et al., 2009), en los suelos de bosque sucedió justo al contrario, presentando éstos un elevado contenido en COP. El CO de los suelos en matorral y cultivo presentó un valor entorno a los $8 \mathrm{~g} \mathrm{~kg}^{-1}$, no mostrando diferencias entre ambos grupos, así como tampoco aparecieron estas diferencias en cuanto a su composición en COP y COM.

En cuanto a la relación con la densidad aparente (DA), las muestras con los valores más altos de DA se relacionaron con los de menor valor de $\mathrm{CO}$, concordando con lo descrito por otros autores (CASTILLO et al., 1997; OHTSUKA et al., 2008; WU et al., 2003).

\subsection{Textura y carbono orgánico en sedimentos}

La textura de las muestras de sedimentos fue parecida a la de los suelos (40-60\% arenas, $35-50 \%$ limos y $<10 \%$ arcillas). A pesar de ser sedimentos muy arenosos, hay que destacar que el porcentaje de limos fue ligeramente superior al de las arenas exceptuando los depósitos de erosión lateralgravitacional donde los valores de arena fueron los más elevados (alrededor de $70 \%$ de arenas en un par de casos) y significativamente distintos a los de las otras formas erosivas. Igualmente, apenas hubo diferencias entre clases en cuanto a las fracciones finas, dejando de lado los depósitos de erosión lateralgravitacional donde los limos se encontraron en menor proporción que en el resto.

El CO total (Figura 4) en las muestras de sedimentos varió entre 2,62-6,81 $\mathrm{g} \mathrm{kg}^{-1}$, correspondiendo la concentración más elevada a las muestras de depósitos de erosión por laboreo y la más baja a los sedimentos procedentes de erosión lateral-gravitacional. Destaca precisamente la elevada concentración de $\mathrm{CO}$ en los sedimentos derivados de la erosión por laboreo, siendo paradójicamente los suelos cultivados los que presentaron valores originales más bajos de $\mathrm{CO}$. La distribución del CO entre las dos fracciones fue bastante parecida en todas las muestras con un $62-76 \%$ del carbono como COM y un 23-37\% como COP, semejante a la encontrada en las muestras de suelos.

\subsection{Relaciones carbón orgánico-textura en suelos y sedimentos}

En las correlaciones efectuadas, se analizaron separadamente las muestras de suelos de matorral y cultivos de las de bosque, para evitar que el elevado contenido en arenas enmascarara los resultados. En los resultados obtenidos 


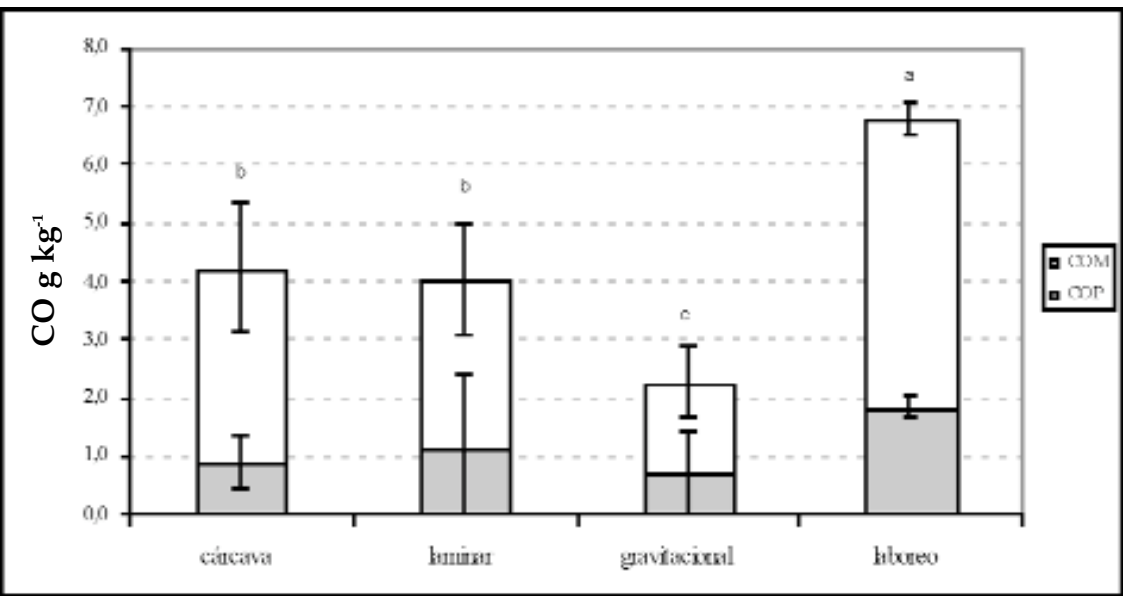

Figura 4. Carbono orgánico repartido en las dos fracciones en los depósitos de las distintas formas erosivas. Diferencias significativas para el valor de $\mathrm{CO}$ total $(\mathrm{COP}+\mathrm{COM})$ marcadas con distintas letras y desviación estándar para COP y MOC.

Figure 4. Organic carbon as a sum of the two fractions in the erosion deposits. Letters indicate significant differences for total organic carbon $(C O P+C O M)$ and standard deviation is given for the $C O P$ and $M O C$ fractions.

(Tabla 1) la fracción textural fina en las muestras de suelo se asoció positivamente al $\mathrm{CO}$ y a la concentración de COM; mientras que las arenas presentaron una relación positiva con la concentración de COP; así como los limos con la concentración de COP, aunque este último en sentido inverso. Es decir, las fracciones más finas se relacionaron positivamente con el $\mathrm{CO}$ y el $\mathrm{COM}$, mientras que las gruesas lo hicieron con el COP. No se estableció ninguna relación entre densidad aparente y textura, ni densidad aparente y carbono. Por otro lado, con los datos de suelo de bosque no se pudo establecer ningún tipo de correlación textura-carbono.

Tabla 1. Coeficientes de correlación de Spearman y significación $\left({ }^{*} \mathrm{p}<0.05 \mathrm{y}{ }^{* *} \mathrm{p}<0.01\right)$ de los mismos para grandes grupos de variables texturales - CO (total, COP y COM) en suelos y sedimentos.

Table 1. Spearman correlation coefficients and significance $\left({ }^{*} p<0.05\right.$ and $\left.{ }^{* *} p<0.01\right)$ between soil texture groupsand organic carbon (total, COP and COM) in soils and sediments.

\begin{tabular}{|ccccccc|}
\hline & \multicolumn{3}{c}{ Suelos $\left(\mathrm{g} \mathrm{kg}^{-1}\right)$} & \multicolumn{3}{c|}{ Sedimentos $(\mathrm{g} \mathrm{kg-1})$} \\
Variable & $C O_{t}$ & COP & COM & $C_{t}$ & COP & COM \\
\hline \% Arenas & 0.015 & $0.516^{*}$ & -0.182 & $-0.552^{* *}$ & $-0.341^{*}$ & $-0.646^{* *}$ \\
\% Limos & -0.024 & $-0.464^{*}$ & 0.174 & $0.562^{* *}$ & $0.324^{*}$ & $0.658^{* *}$ \\
\% Arcillas & $0.653^{* *}$ & 0.152 & $0.587^{*}$ & $0.551^{* *}$ & $0.422^{*}$ & $0.616^{* *}$ \\
\hline
\end{tabular}


De modo muy parecido, en las muestras de sedimentos las correlaciones relacionaron las fracciones texturales finas al contenido en $\mathrm{CO}, \mathrm{COM}$ y COP, mientras que los resultados fueron en sentido inverso para la fracción textural arenas.

\subsection{Enriquecimiento de carbono orgánico y textura}

En general, los depósitos de todos los procesos erosivos se encontraron ligeramente enriquecidos en arcilla (de mayor a menor valor: laminar> cárcava $>$ laboreo $>$ lateral-gravitacional) correspondiendose bastante bien con la naturaleza física del proceso (Tabla 2).

Las muestras de sedimentos presentaron concentraciones en $\mathrm{CO}$ mucho más bajas que los suelos originales de la cuenca, lo cual se constató con una

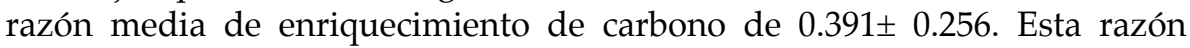
concuerda bastante bien con las encontradas en otros trabajos a escala de cuenca, también por debajo de la unidad: 0.77 (BOIX-FAYOS et al., 2009); 0.65 (RHOTON et al., 2006); 0.43-1.83 (80\% de los datos) (AVNIMELECH \& MCHENRY, 1984). La escala de trabajo condiciona los valores de las razones de enriquecimiento. Así, a escalas de mayor resolución (o detalle) los procesos son más selectivos eliminando las partículas más finas y superficiales de suelo, más enriquecidas en carbono (valores entre 0.8 y 1.8 de: STRICKLAND et al., 2005; SCHIETTECATTE et al., 2008a; POLYAKOV \& LAL, 2004), mientras que a escalas más gruesas los depósitos de sedimentos totalizan eventos de distintas características con menor selectividad en el tamaño de las partículas e incorporan no sólo sedimentos de transporte en suspensión (más finos) sino sedimentos más gruesos como carga de fondo que presentan menores concentraciones de CO. Sin embargo, ocasionalmente se han encontrado valores de RE muy elevados, hasta 4, en cuencas de tamaño mediano (0.02-0.18 $\left.\mathrm{km}^{2}\right)$ (WEIGAND et al., 1998). En el presente trabajo, la baja razón de enriquecimiento obtenida puede ser consecuencia del predominio de formas erosivas poco selectivas en el arranque y el transporte de los sedimentos junto con elevadas tasas de mineralización debido a la elevada inestabilidad y exposición de los sedimentos en sus respectivos depósitos.

Llama la atención el hecho de que la desviación de la RE de los datos fue muy elevada (0.256) en relación al valor de la media (0.391), lo cual refleja la gran variabilidad de valores de $\mathrm{CO}$ en las muestras recogidas y permite intuir una diferenciación de erosión de $\mathrm{CO}$ entre procesos erosivos, pues las desviaciones dentro de los grupos fueron más bajas (del orden de $\sim 0,06$ ). 
La hipótesis de partida se confirma con los datos obtenidos dada las diferencias significativas observadas en la concentración de $\mathrm{CO}$ entre los distintos procesos erosivos estudiados. Se obtuvieron, sin embargo, concentraciones de CO más bajas que lo esperado en el proceso de erosión laminar comparados con los obtenidos en la erosión en cárcavas (Tabla 3). Uno de los motivos de estos resultados podría ser metodológico al ser difícil localizar ubicaciones claras de depósitos de erosión laminar en el barranco dada su elevada actividad erosiva.

Tabla 2. Razones de enriquecimiento de arena, limo y arcilla. Diferentes letras en las columnas indican diferencias significativas. Nivel de significación del 95\%.

Table 2. Enrichment ratios for sand, silt and clay. Different letters indicate significant differences within columns ( $95 \%$ confidence interval).

\begin{tabular}{|cccc|}
\hline Forma erosiva & Arenas & Limos & Arcillas \\
\hline Cárcava & $0.906 \pm 0.297^{\mathrm{ab}}$ & $1.033 \pm 0.217^{\mathrm{a}}$ & $1.435 \pm 0.520^{\mathrm{a}}$ \\
Laminar & $0.786 \pm 0.251^{\mathrm{b}}$ & $1.157 \pm 0.218^{\mathrm{a}}$ & $1.634 \pm 0.514^{\mathrm{a}}$ \\
Lateral-gravitacional & $1.174 \pm 0.227^{\mathrm{a}}$ & $0.802 \pm 0.178^{\mathrm{b}}$ & $1.045 \pm 0.596^{\mathrm{a}}$ \\
Laboreo & $0.934 \pm 0.067^{\mathrm{ab}}$ & $1.049 \pm 0.025^{\mathrm{a}}$ & $1.117 \pm 0.280^{\mathrm{a}}$ \\
\hline
\end{tabular}

Tabla 3. Razones de enriquecimiento de carbono orgánico. Diferentes letras en las columnas indican diferencias significativas. Nivel de significación del 95\%.

Table 3. Enrichment ratios for organic carbon. Different letters in columns indicate significant differences (95\% confidence interval).

\begin{tabular}{|cccc|}
\hline Forma erosiva & $\mathrm{CO}_{t}$ & $C O P\left(\mathrm{~g} \mathrm{~kg}^{-1}\right)$ & $C O M\left(\mathrm{~g} \mathrm{~kg}^{-1}\right)$ \\
\hline Cárcava & $0.482 \pm 0.092^{\mathrm{b}}$ & $0.288 \pm 0.179^{\mathrm{b}}$ & $0.589 \pm 0.114^{\mathrm{b}}$ \\
Laminar & $0.195 \pm 0.068^{\mathrm{c}}$ & $0.094 \pm 0.072^{\mathrm{c}}$ & $0.346 \pm 0.133^{\mathrm{c}}$ \\
Lateral-gravitacional & $0.111 \pm 0.062^{\mathrm{d}}$ & $0.057 \pm 0.074^{\mathrm{c}}$ & $0.192 \pm 0.052^{\mathrm{d}}$ \\
Laboreo & $0.885 \pm 0.033^{\mathrm{a}}$ & $0.992 \pm 0.111^{\mathrm{a}}$ & $0.851 \pm 0.040^{\mathrm{a}}$ \\
\hline
\end{tabular}

Finalmente, se relacionaron las RE en carbono orgánico total y RE en finos (limo fino y arcilla) en un gráfico de dispersión (Figura 5). Al analizar los procesos erosivos por separado aparecen unas tendencias claras: todos los depósitos, a excepción de los de erosión por laboreo, presentan una relación positiva entre las RE de fracciones texturales finas (limo y arcillas) y enriquecimiento de CO. Si bien los depósitos de erosión por laboreo presentan la tendencia contraria, interpretándose de escasa validez este resultado debido al escaso número de muestras de este grupo y la poca 
variación de RE entre ellas, necesitándose de mayor número de muestras para poder confirmar o desmentir esta última tendencia mediante análisis de regresión.

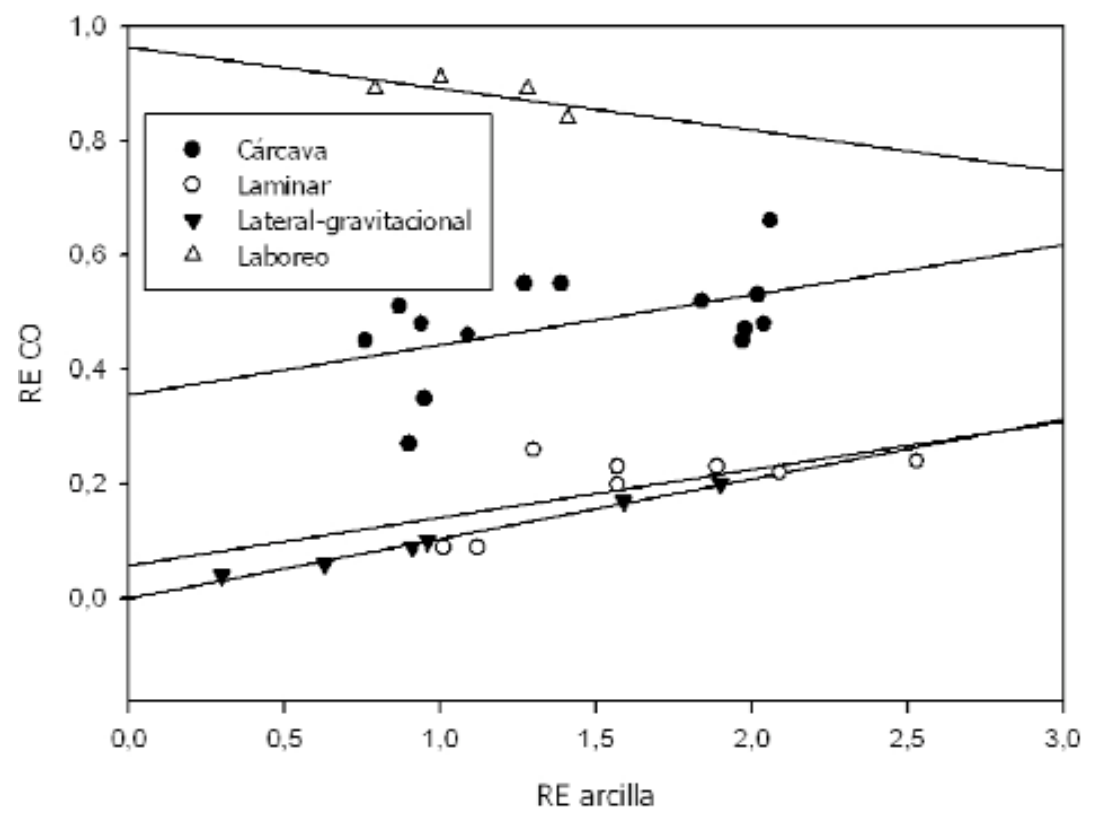

Figura 5. Relación entre RE de CO y RE de arcilla en los cuatro procesos erosivos. Figure 5. Relationship between RE for CO and RE for clay for the four erosion processes.

En cualquier caso, las tendencias en los otros grupos confirman los resultados de los análisis de correlación anteriores que indicaban una asociación positiva entre movimiento de partículas finas y movilización de $\mathrm{CO}$. A pesar de las bajas razones de $\mathrm{CO}$ en los depósitos analizados en general, el empobrecimiento es menor en aquellos que se encuentran más enriquecidos en partículas finas. Estos resultados se asemejan a los encontrados en otros trabajos (JIN et al., 2009; RHOTON et al., 2006).

\subsection{Procesos erosivos y movilización de carbono orgánico}

A modo general se puede decir que es en los procesos erosivos menos selectivos en cuanto al arranque y transporte de material donde menor 
concentración de carbono presentaron los sedimentos. La secuencia de procesos erosivos a creciente concentración de $\mathrm{CO}$ en los sedimentos fue: erosión lateral/gravitacional < erosión en laminar < erosión en cárcava $<$ erosión por laboreo.

Estos resultados resultan coherentes con los de numerosos autores que afirman que la erosión por laboreo moviliza cantidades importantes de carbono orgánico al afectar a la capa más superficial del suelo (DEL GALDO et al., 2003; MORARI et al., 2006; POST \& KWON, 2000). Asimismo los valores en depósitos de cárcava se encuentran en consonancia con los resultados de SCHIETTECATTE et al. (2008a) que en ensayos de laboratorio atribuyeron los bajos contenidos en carbono de la erosión concentrada a la no selectividad de este proceso y a que afecta a mayor profundidad del perfil edáfico que la erosión laminar.

A nivel textural, tan solo los sedimentos de erosión lateral-gravitacional se distinguieron del resto con una textura enriquecida en arenas y empobrecida en limos y contuvieron además los valores más bajos de CO, al afectar al perfil de suelo entero e incluso al regolito (en este caso areniscas) y movilizar partículas de mayor tamaño.

\section{Conclusiones}

Todos los depósitos sedimentarios analizados presentaron un empobrecimiento en $\mathrm{CO}$ con respecto a los suelos de la cuenca. El contenido en $\mathrm{CO}$ de éstos depósitos varió en función del proceso erosivo que los originó; de este modo, se constató que procesos no selectivos como la erosión lateralgravitacional o la erosión en cárcavas, que movilizan material de todo el perfil edáfico y hasta del regolito, presentaron concentraciones de $\mathrm{CO}$ menores que procesos que afectan solamente a la parte superficial del perfil, como la erosión por laboreo. La proporción de COP y COM en suelos y sedimentos fue muy parecida aunque las razones de enriquecimiento de COM fueron ligeramente más altas que las de COP en la mayoría de los casos.

Por lo general, los sedimentos de la cuenca presentaron una textura enriquecida en limo y arcilla y empobrecida en arena, con respecto a los suelos originales. A pesar de que las fracciones finas se relacionaron con el contenido en $\mathrm{CO}$ los sedimentos no resultaron enriquecidos en CO. Estos resultados se atribuyen a los procesos de mineralización en parte del $\mathrm{CO}$ originalmente vinculado a las fracciones finas debida a la elevada actividad erosiva presente en las conexiones ladera-cauce y la escasa presencia de zonas de deposición estables. 


\section{Agradecimientos}

Este trabajo ha sido financiado por los proyectos ERCO (CGL-200762590 / BTE) del Ministerio de Ciencia e Innovación y el proyecto PROBASE (CGL2006-11619). La primera autora tiene el apoyo económico de una ayuda FPI del Ministerio de Ciencia e Innovación (BES-2008-002379). Se agradece la colaboración de los técnicos de laboratorio del grupo de erosión y conservación de suelos del CEBAS-CSIC.

\section{Referencias}

AVNIMELECH, Y. \& MCHENRY, J. R. (1984). Enrichment of transported sediments with organic carbon, nutrients and clay. Soil Science Society of America Journal 48(2): 259-266.

BERHE, A. A., HARDEN, J. W., TORN, M. S. \& HARTE, J. (2008). Linking soil organic matter dynamics and erosion-induced terrestrial carbon sequestration at different landform positions. Journal of Geophysical Research 113(4): art. no. G04039.

BERHE, A. A., HARTE, J., HARDEN, J. W. \& TORN, M. S. (2007). The significance of the erosion-induced terrestrial carbon sink. BioScience 57(4): 337-346.

BOIX-FAYOS, C., DE VENTE, J., ALBALADEJO, J. \& MARTÍNEZ-MENA, M. (2009). Soil carbon erosion and stock as affected by land use changes at the catchment scale in Mediterranean ecosystems. Agriculture, Ecosystems \& Environment 133(1-2): 75-85.

CAMBARDELLA, C.A. \& ELLIOT, E.T. (1992). Particulate organic matter changes across a grassland cultivation sequence. Soil Science Society of America Journal 56(3): 777-783.

CASTILLO, V. M., MARTINEZ-MENA, M. \& ALBALADEJO, J. (1997). Runoff and Soil Loss Response to Vegetation Removal in a Semiarid Environment. Soil Science Society of America Journal 61(4): 1116-1121.

DE VENTE, J., POESEN, J., ARABKHEDRI, M. \& VERSTRAETEN, G. (2007). The sediment delivery problem revisited. Progress in Physical Geography 31(2): 155-178.

DEL GALDO, I., SIX, J., PERESSOTTI, A. \& COTRUFO, M. F. (2003). Assessing the impact of land-use change on soil $\mathrm{C}$ sequestration in agricultural soils by means of organic matter fractionation and stable $\mathrm{C}$ isotopes. Global Change Biology 9(8): 1204-1213.

GREGORICH, E. G., GREER, K. J., ANDERSON, D. W. \& LIANG, B. C. (1998). Carbon distribution and losses: Erosion and deposition effects. Soil and Tillage Research 47(3-4): 291-302. 
HAREGEWEYN, N., POESEN, J., DECKERS, J., NYSSEN, J., HAILE, M., GOVERS, G., VERSTRAETEN, G. \& MOEYERSONS, J. (2008). Sedimentbound nutrient export from micro-dam catchments in Northern Ethiopia. Land Degradation \& Development 19(2): 136-152.

JACINTHE, P. A., LAL, R., OWENS, L. B. \& HOTHEM, D. L. (2004). Transport of labile carbon in runoff as affected by land use and rainfall characteristics. Soil and Tillage Research 77(2): 111-123.

JIN, K., CORNELIS, W. M., GABRIELS, D., BAERT, M., WU, H. J., SCHIETTECATTE, W., CAI, D. X., DE NEVE, S., JIN, J. Y., HARTMANN, R. \& HOFMAN, G. (2009). Residue cover and rainfall intensity effects on runoff soil organic carbon losses. Catena 78(1): 81-86.

LAL, R. (2003). Soil erosion and the global carbon budget. Environment International 29(4): 437-450.

LAL, R. (2002). Soil carbon dynamics in cropland and rangeland. Environmental Pollution 116(3): 353-362.

LAL, R. (ed) (2001). Assessment Methods for Soil Carbon. Advances in Soil Science. CRC Press, 403-416 pp. Boca Raton, Fl.

LIU, S. G., BLISS, N., SUNDQUIST, E. \& HUNTINGTON, T. G. (2003). Modeling carbon dynamics in vegetation and soil under the impact of soil erosion and deposition. Global Biogeochemical Cycles 17(2): 1074, doi:10.1029/2002GB002010.

MARTINEZ-MENA, M., LÓPEZ, J., ALMAGRO, M., BOIX-FAYOS, C. \& ALBALADEJO, J. (2008). Effect of water erosion and cultivation on the soil carbon stock in a semiarid area of South-East Spain. Soil and Tillage Research 99(1): 119-129.

MORARI, F., LUGATO, E., BERTI, A. \& GIARDINI, L. (2006). Long-term effects of recommended management practices on soil carbon changes and sequestration in north-eastern Italy. Soil Use and Management 22(1): 71-81.

OHTSUKA, T., HIROTA, M., ZHANG, X., SHIMONO, A., SENGA, Y., DU, M., YONEMURA, S., KAWASHIMA, S. \& TANG, Y. (2008). Soil organic carbon pools in alpine to nival zones along an altitudinal gradient (4400$5300 \mathrm{~m}$ ) on the Tibetan Plateau. Polar Science 2(4): 277-285.

OWENS, L. B., MALONE, R. W., HOTHEM, D. L., STARR, G. C. \& LAL, R. (2002). Sediment carbon concentration and transport from small watersheds under various conservation tillage practices. Soil and Tillage Research 67(1): 65-73.

POLYAKOV, V. O. \& LAL, R. (2004). Soil erosion and carbon dynamics under simulated rainfall. Soil Science 169(8): 590-599.

POST, W. M. \& KWON, K. C. (2000). Soil carbon sequestration and land-use change: processes and potential. Global Change Biology 6(3): 317-327.

QUINTON, J. N., CATT, J. A., WOOD, G. A. \& STEER, J. (2006). Soil carbon losses by water erosion: Experimentation and modeling at field and 
national scales in the UK. Agriculture, Ecosystems \& Environment 112(1): 87102.

RHOTON, F. E., EMMERICH, W. E., GOODRICH, D. C., MILLER, S. N. \& MCCHESNEY, D. S. (2006). Soil Geomorphological Characteristics of a Semiarid Watershed: Influence on Carbon Distribution and Transport. Soil Science Society of America Journal 70(5): 1532-1540.

RITCHIE, J. C., MCCARTY, G. W., VENTERIS, E. R. \& KASPAR, T. C. (2007). Soil and soil organic carbon redistribution on the landscape. Geomorphology 89(1-2): 163-171.

RODRIGUEZ, A. R., GUERRA, A., ARBELO, C., MORA, J. L., GORRIN, S. P. \& ARMAS, C. (2004). Forms of eroded soil organic carbon in andosols of the Canary Islands (Spain). Geoderma 121(3-4): 205-219.

ROOSE, E. J. L., R.; FELLER, C.; BARTHÈS, B.; STEWART, B.A. (2006), Soil Erosion and Carbon Dynamics, CRC Press, 376 pp, Boca Raton, FL.

SCHIETTECATTE, W., GABRIELS, D., CORNELIS, W. M. \& HOFMAN, G. (2008a). Enrichment of organic carbon in sediment transport by interrill and rill erosion processes. Soil Science Society of America Journal 72(1): 5055 .

SCHIETTECATTE, W., GABRIELS, D., CORNELIS, W. M. \& HOFMAN, G. (2008b). Impact of deposition on the enrichment of organic carbon in eroded sediment. Catena 72(3): 340-347.

SCHLESINGER, W. H. \& MELACK, J. M. (1981). Transport of organic carbon in the world's rivers. Tellus 33(2): 172-187.

SMITH, S. V., BULLOCK, S. H., HINOJOSA-CORONA, A., FRANCOVIZCAINO, E., ESCOTO-RODRIGUEZ, M., KRETZSCHMAR, T. G., FARFAN, L. M. \& SALAZAR-CESENA, J. M. (2007). Soil erosion and significance for carbon fluxes in a mountainous Mediterranean-climate watershed. Ecological Applications 17(5): 1379-1387.

SMITH, S. V., RENWICK, W. H., BUDDEMEIER, R. W. \& CROSSLAND, C. J. (2001). Budgets of soil erosion and deposition for sediments and sedimentary organic carbon across the conterminous United States. Global Biogeochemical Cycles 15(3): 697-707.

STALLARD, R. F. (1998). Terrestrial sedimentation and the carbon cycle: Coupling weathering and erosion to carbon burial. Global Biogeochemical Cycles 12(2): 231-257.

STOLTENBERG, N. L. \& WHITE, J. L. (1953). Selective Loss of Plant Nutrients by Erosion. Soil Science Society of America Journal 17(4): 406-410.

STRICKLAND, T. C., TRUMAN, C. C. \& FRAUENFELD, B. (2005). Variable rainfall intensity effects on carbon characteristics of eroded sediments from two coastal plain ultisols in Georgia. Journal of Soil and Water Conservation 60(3): 142-148. 
MOVILIZACIÓN DE CARBONO ORGÁNICO POR DISTINTOS PROCESOS EROSIVOS...

VAN OOST, K., GOVERS, G., QUINE, T. A., HECKRATH, G., OLESEN, J. E., DE GRYZE, S. \& MERCKX, R. (2005). Landscape-scale modeling of carbon cycling under the impact of soil redistribution: The role of tillage erosion. Global Biogeochemical Cycles 19(4): GB4014, doi:10.1029/2005GB002471.

WALLING, D. E. (1983). The sediment delivery problem. Journal of Hydrology 65(1-3): 209-237.

WEI, J. B., XIAO, D. N., ZENG, H. \& FU, Y. K. (2008). Spatial variability of soil properties in relation to land use and topography in a typical small watershed of the black soil region, northeastern China. Environmental Geology 53(8): 1663-1672.

WEIGAND, S., SCHIMMACK, W. \& AUERSWALD, K. (1998). The enrichment of 137Cs in the soil loss from small agricultural watersheds. Journal of Plant Nutrition and Soil Science 161(4): 479-484.

WU, H. B., GUO, Z. T. \& PENG, C. H. (2003). Land use induced changes of organic carbon storage in soils of China. Global Change Biology 9(3): 305315.

YEOMANS, J.C. \& BREMNER, J.M. (1988). A rapid and precise method for routine determination of organic carbon in soil. Communications in Soil Science \& Plant Analysis 19(13): 1467-1476.

YOO, K. (2005). Erosion of upland hillslope soil organic carbon: Coupling field measurements with a sediment transport model. Global biogeochemical cycles 19(3): no. art. GB3003.

ZHANG, J. H., QUINE, T. A., NI, S. J. \& GE, F. L. (2006). Stocks and dynamics of SOC in relation to soil redistribution by water and tillage erosion. Global Change Biology 12(10): 1834-1841. 